N. Srocka, P. Mrowiński, J. Große, M. von Helversen, Tobias Heindel, Sven Rodt, Stephan Reitzenstein

\title{
Deterministically fabricated quantum dot single-photon source emitting indistinguishable photons in the telecom O-band
}

Journal article | Published version

This version is available at https://doi.org/10.14279/depositonce-11271

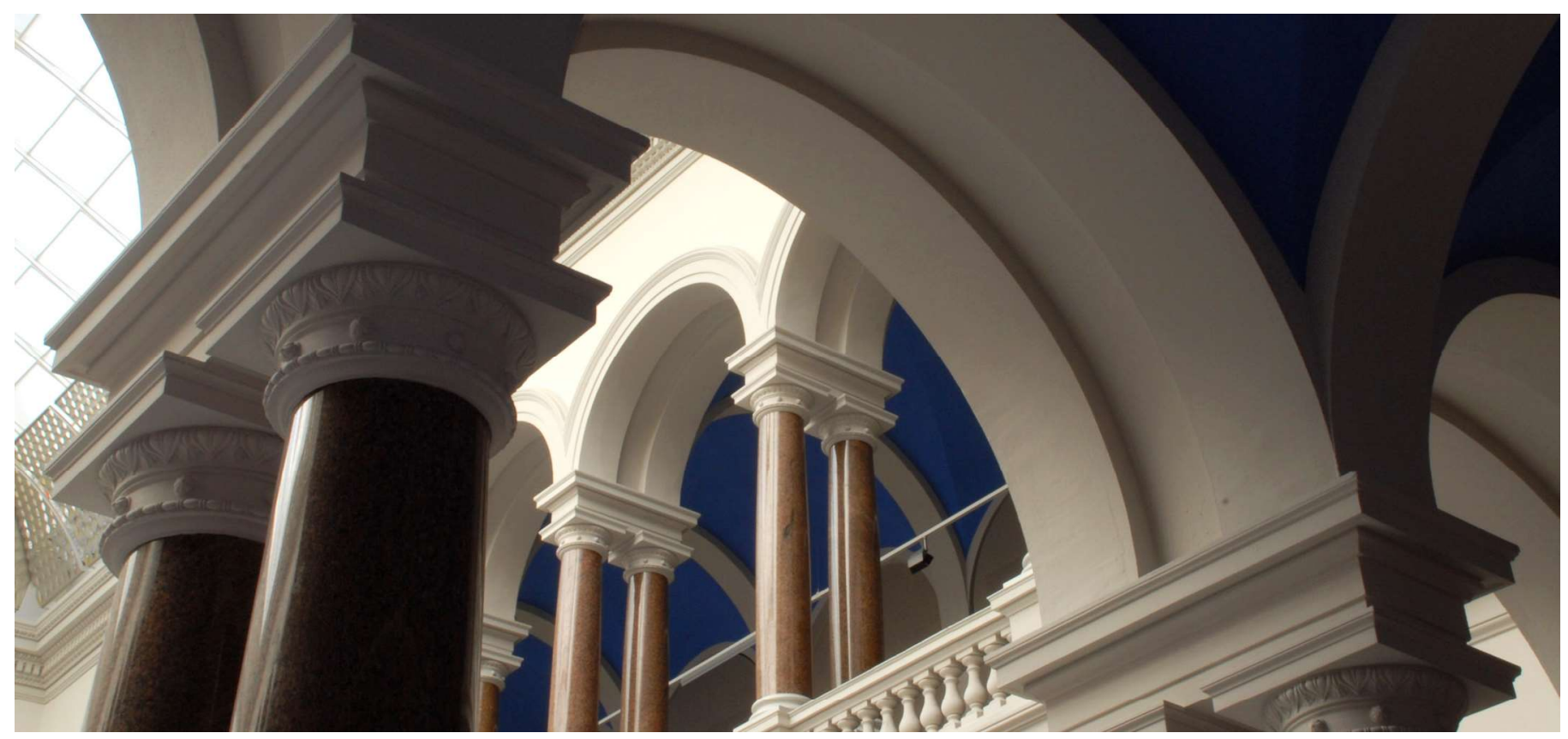

This article may be downloaded for personal use only. Any other use requires prior permission of the author and AIP Publishing. This article appeared in

Srocka, N., Mrowiński, P., Große, J., von Helversen, M., Heindel, T., Rodt, S. \& Reitzenstein, S. (2020). Deterministically fabricated quantum dot single-photon source emitting indistinguishable photons in the telecom O-band. Applied Physics Letters, 116(23), 231104. https://doi.org/10.1063/5.0010436

and may be found at https://doi.org/10.1063/5.0010436. 


\section{Deterministically fabricated quantum dot single-photon source emitting indistinguishable photons in the telecom 0- band ()$^{2}$}

Cite as: Appl. Phys. Lett. 116, 231104 (2020); https://doi.org/10.1063/5.0010436

Submitted: 10 April 2020. Accepted: 16 May 2020. Published Online: 09 June 2020

N. Srocka, P. Mrowiński, J. Große, M. von Helversen, (D) T. Heindel, S. Rodt, and (D) S. Reitzenstein

COLLECTIONS

EP This paper was selected as an Editor's Pick
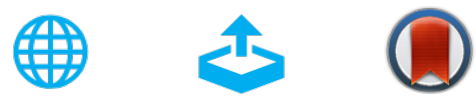

View Online

Export Citation

\section{ARTICLES YOU MAY BE INTERESTED IN}

Progress in quantum-dot single photon sources for quantum information technologies: $A$ broad spectrum overview

Applied Physics Reviews 7, 021309 (2020); https://doi.org/10.1063/5.0010193

Coherence and indistinguishability of highly pure single photons from non-resonantly and resonantly excited telecom C-band quantum dots

Applied Physics Letters 115, 023103 (2019); https://doi.org/10.1063/1.5095196

Superconducting quantum many-body circuits for quantum simulation and computing Applied Physics Letters 116, 230501 (2020); https://doi.org/10.1063/5.0008202

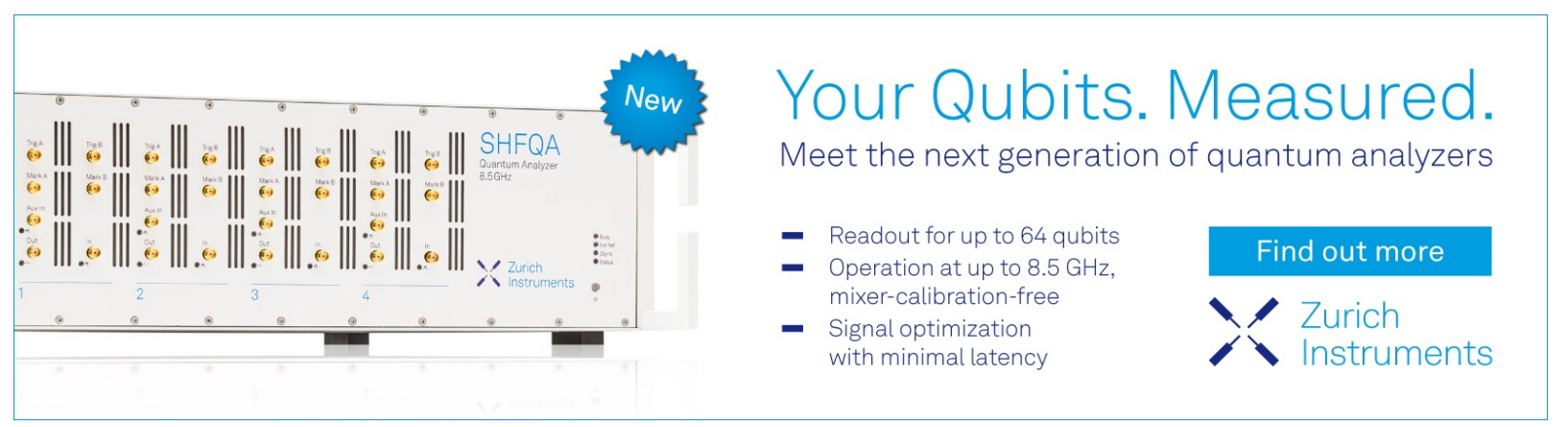




\title{
Deterministically fabricated quantum dot single-photon source emitting indistinguishable photons in the telecom O-band 주
}

\author{
Cite as: Appl. Phys. Lett. 116, 231104 (2020); doi: 10.1063/5.0010436 \\ Submitted: 10 April 2020 - Accepted: 16 May 2020 • \\ Published Online: 9 June 2020

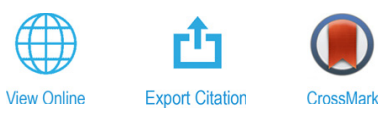

N. Srocka, ${ }^{1}$ P. Mrowiński, ${ }^{1,2}$ J. Große, ${ }^{1}$ M. von Helversen, ${ }^{1}$ T. Heindel, ${ }^{1}$ (D S. Rodt, ${ }^{1}$ and S. Reitzenstein ${ }^{1, a)}$ (iD)

\begin{abstract}
AFFILIATIONS
'Institut für Festkörperphysik, Technische Universität Berlin, Hardenbergstraße 36, 10623 Berlin, Germany

${ }^{2}$ Laboratory for Optical Spectroscopy of Nanostructures, Department of Experimental Physics, Wrocław University of Technology, Wybrzeże Wyspiańskiego 27, Wrocław, Poland

${ }^{a)}$ Author to whom correspondence should be addressed: stephan.reitzenstein@physik.tu-berlin.de
\end{abstract}

\begin{abstract}
In this work, we develop and study single-photon sources based on InGaAs quantum dots (QDs) emitting in the telecom O-band. Quantum devices are fabricated using in situ electron beam lithography in combination with thermocompression bonding to realize a backside gold mirror. Our structures are based on InGaAs/GaAs heterostructures, where the QD emission is redshifted toward the telecom O-band at $1.3 \mu \mathrm{m}$ via a strain-reducing layer. QDs pre-selected by cathodoluminescence mapping are embedded into mesa structures with a backside gold mirror for enhanced photon-extraction efficiency. Photon-autocorrelation measurements under pulsed non-resonant wetting-layer excitation are performed at temperatures up to $40 \mathrm{~K}$, showing pure single-photon emission, which makes the devices compatible with stand-alone operation using Stirling cryocoolers. Using pulsed p-shell excitation, we realize single-photon emission with a high multiphoton suppression of $\mathrm{g}^{(2)}(0)=0.027 \pm 0.005$, an as-measured two-photon interference visibility of $(12 \pm 4) \%$, a post-selected visibility of $(96 \pm 10) \%$, and an associated coherence time of $(212 \pm 25)$ ps. Moreover, the structures show an extraction efficiency of $\sim 5 \%$, which is comparable to values expected from numeric simulations of this photonic structure. Further improvements of our devices will enable implementations of quantum communication via optical fibers.
\end{abstract}

Published under license by AIP Publishing. https://doi.org/10.1063/5.0010436

Single photons, often referred to as flying qubits, are key resources in the field of photonic quantum technology and enable, for instance, the distribution of quantum information over large distances. ${ }^{1,2}$ Moving beyond simple point-to-point quantum key distribution protocols, such as BB84, ${ }^{3}$ the quantum nature of single photons in terms of high photon indistinguishability becomes important. In fact, high photon indistinguishability is required in large-scale quantum networks based, e.g., on entanglement distribution via Bell state measurements. Moreover, single photon emitters have to comply with the existing optical fiber infrastructure for long-haul communication in the telecommunication O-band at $1.3 \mu \mathrm{m}$ or in the C-band at $1.55 \mu \mathrm{m} .{ }^{4-14}$ Therefore, besides a robust device concept with excellent quantum properties, spectral matching to one of the two telecom bands is needed to enable fiber-based quantum communication of large distances. Despite significant progress, ${ }^{6-9,13-20}$ it is still a great challenge to fulfill these stringent requirements with on-demand quantum dot (QD)-based single-photon sources (SPSs). In particular, compared to their short wavelength counterparts, InGaAs QDs emitting in the telecom bands suffer from low indistinguishability with maximum visibilities of $(18 \pm 1) \%$ without post-selection and $(67 \pm 2) \%$ with post-selection in the case of $1.3 \mu \mathrm{m}$ cavity-coupled QDs. ${ }^{8}$ For $1.55 \mu \mathrm{m}$ QDs, the indistinguishability has so far only be studied under CW excitation, leading to a post-selected visibility of $(89.4 \pm 10.9) \% .^{21}$

In this work, we address the above-mentioned requirements within a deterministic device technology. We transfer and optimize technology concepts, which allowed for high-quality emission from semiconductor QDs in the spectral range below $1 \mu \mathrm{m}$, to deterministically realize bright QD single-photon sources (SPSs) emitting in the telecom O-band. The sources are based on self-assembled InGaAs/ GaAs QDs grown by metal organic chemical vapor deposition (MOCVD), where a strain-reducing layer (SRL) is applied to shift the emission wavelength to the telecom O-band. ${ }^{10,22-24}$ Using in situ electron beam lithography (EBL), such QDs are deterministically 
integrated into nanophotonic structures. Here, low-temperature cathodoluminescence (CL) spectroscopy is used to pre-select suitable QDs based on their emission wavelengths and brightness, before micromesas, allowing for enhanced photon-extraction efficiency, are patterned with an alignment accuracy ${ }^{25}$ of $30-40 \mathrm{~nm}$ by in situ EBL in the same system. The realized QD-micromesas with a backside gold mirror are designed to enable broadband enhancement of photon-extraction efficiency. In this way, we realize high-quality telecom O-band SPSs with strong suppression of multi-photon emission events up to $40 \mathrm{~K}$. Moreover, by means of Hong-Ou-Mandel (HOM) experiments under pulsed excitation, we determine a two-photon interference (TPI) visibility of $96 \%$ under temporal post-selection and an applicationrelevant value of $12 \%$ without post-selection.

We developed an advanced flip-chip based-processing concept for telecom SPSs in which a single-QD device is fabricated within three main processing steps. First, a GaAs semiconductor heterostructure is grown by MOCVD including a single layer of self-assembled InGaAs QDs. Then, a thin membrane of this structure is transferred on a gold mirror by thermocompression bonding and wet-chemical etching, before single QDs inside this membrane are deterministically integrated into mesa structures by means of in situ EBL.

The epitaxial growth starts with a $200 \mathrm{~nm}$ thick GaAs buffer on top of an n-doped GaAs (100) substrate to enable a high-quality epitaxial surface. Then, two $\mathrm{Al}_{0.90} \mathrm{Ga}_{0.10}$ As layers of $1 \mu \mathrm{m}$ and $100 \mathrm{~nm}$ thicknesses are deposited. These two layers are separated by a $2 \mu \mathrm{m}$-thick GaAs spacer layer, which allows for a well-controlled twostep wet-chemical etching procedure to remove the initial substrate. All these layers act as etch-stop and sacrificial layers and will be removed during the flip-chip post-growth processing. ${ }^{26,27}$ The growth resumes with $637 \mathrm{~nm}$ GaAs, 1.5 monolayers of $\operatorname{In}_{0.7} \mathrm{Ga}_{0.3}$ As followed by an 0.5 monolayer GaAs flush to realize the QD layer, $5.5 \mathrm{~nm} \mathrm{InGaAs}$, whereby the In content is linearly decreased from $30 \%$ to $10 \%$, forming the SRL, and a final GaAs capping layer of $242 \mathrm{~nm}$, which completes the layer structure. The described layer design is depicted in Fig. 1(a).

To enable thermocompression gold bonding, a $250 \mathrm{~nm}$ thick gold layer is deposited on the as-grown heterostructure sample as well as on a bare piece of the GaAs (100) substrate acting as a host substrate. The sample is bonded to the host substrate (gold facing gold) by applying a pressure of $6 \mathrm{MPa}$ and a temperature of approximately $600 \mathrm{~K}$ for four hours. Subsequent wet-chemical etching is performed

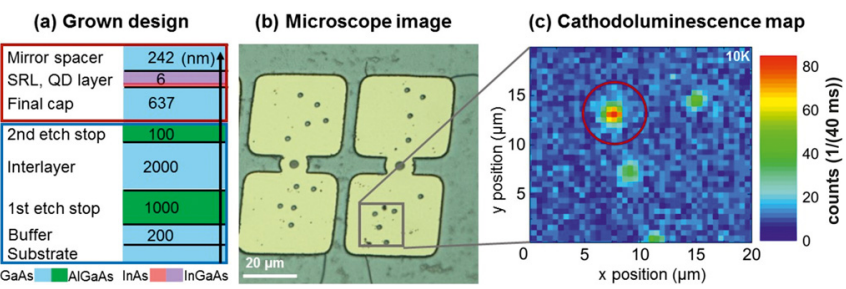

FIG. 1. (a) Schematic epitaxial layer design. Layers in the blue box are etch-stop/ sacrificial layers that are removed during the post-growth processing. After a flipchip process, the layers in the red box are bonded up-side-down onto a gold mirror. For this reason, the given layer names refer to their final device functionality. (b) Optical microscopy image of the final device. Four to five mesas are processed per mapping field. (c) Low temperature CL map recorded after full processing (integration time/pixel: $40 \mathrm{~ms}$; spectral range depicted: target wavelength $\pm 1 \mathrm{~nm}$ ). The red circle marks the QD-micromesa investigated in the following. in two steps. First, the $300 \mu \mathrm{m}$ thick GaAs substrate is lifted off with a fast etching solution $\left(\mathrm{H}_{2} \mathrm{O}_{2} / \mathrm{NH}_{3}, 10: 1\right)$, where the etching stops at the first $\mathrm{Al}_{0.97} \mathrm{Ga}_{0.03} \mathrm{As}$ layer. After an $\mathrm{HCl}$ dip removing this etch-stop layer, the following GaAs layer is removed by a different etchant (citric acid/ $\mathrm{H}_{2} \mathrm{O}_{2}, 4: 1$ ). Here, the less aggressive etchant was chosen in favor of an improved surface roughness over a high etch rate. A final $\mathrm{HCl}$ dip leaves only a thin GaAs membrane of $885 \mathrm{~nm}$, including the active QD layer, with gold being bonded to the host GaAs substrate. On the cleaned sample, $250 \mathrm{~nm}$ of AR-P 6200 electron-beam resist are spin coated in preparation for the lithography step.

In the subsequent main processing step, single pre-selected QDs are integrated deterministically into micromesas to enhance their photon-extraction efficiency. ${ }^{28,29}$ QD pre-selection is performed by CL mapping over $(20 \times 20) \mu \mathrm{m}^{2}$ sample areas at $10 \mathrm{~K}$. Immediately after that, mesa patterns are written into the resist at the chosen QD positions by EBL in the same system at $10 \mathrm{~K}$. During the subsequent resist development, the mapping area is cleared and only EBL-patterned areas remain, acting as masks in the subsequent inductively coupledplasma reactive-ion etching of QD mesas. We refer to Refs. 25 and 30 for further details of the in situ EBL technique. Figure 1(b) shows an optical microscopy image, and panel (c) presents a CL map of deterministic QD mesa structures. The CL map in Fig. 1(c) was taken with the same settings as for the pre-selection step, clearly showing the fabrication of deterministic QD-micromesas.

Optical and quantum optical characterization of the QD mesas is performed by means of microphotoluminescence $(\mu \mathrm{PL}), \mu \mathrm{PL}$-excitation $(\mu \mathrm{PLE})$, and photon-correlation spectroscopy, respectively. The sample is mounted in a He-flow cryostat with temperature control in the range of $10 \mathrm{~K}-40 \mathrm{~K}$. Excitation is provided by a CW laser and tunable pulsed lasers focused on the sample by a microscope objective [numerical aperture (NA) $=0.4$ ]: a continuous-wave diode laser $(785 \mathrm{~nm})$ and a tunable laser, providing ps-pulses at a repetition rate of $80 \mathrm{MHz}$. The photoluminescence signal is collected with the same objective, spectrally resolved in a grating spectrometer (spectral resolution $\sim 0.05 \mathrm{~nm}$ ) and either detected using a liquid nitrogencooled InGaAs-array detector or coupled into a fiber-based Hanbury-Brown and Twiss (HBT) or a Hong-Ou-Mandel (HOM) setup. For HBT and HOM measurements, the photons are detected using two superconducting nanowire single photon detectors (SNSPDs), each with a temporal resolution of $\sim 50$ ps and a detection efficiency of about $80 \%$ at $1310 \mathrm{~nm}$.

First, temperature-dependent $\mu$ PL spectra and photon autocorrelation histograms were recorded for the selected deterministic photonic microstructure marked in Fig. 1(c). Corresponding $\mu$ PL spectra are presented in Fig. 2(a) for temperatures from $10 \mathrm{~K}$ to $40 \mathrm{~K}$. Three excitonic states-exciton (X), biexciton (XX), and charged exciton (CX)—of the single-QD mesa are identified by excitation-power- and polarizationdependent $\mu \mathrm{PL}$ studies (see the supplementary material-Fig. S1). A temperature-induced red shift is observed together with a notably stable single-QD emission up to $40 \mathrm{~K}$, reflecting a rather deep carrier confinement in this type of QD. The temperature stability is an important aspect regarding the development of stand-alone SPS operated by Stirling cryocoolers with a base temperature between $30 \mathrm{~K}$ and $40 \mathrm{~K}$ (depending on the manufacturer). ${ }^{31-33}$ The brightest CX emission line is further examined in the HBT setup to investigate the quantum nature of emission. Here, we obtain a high multi-photon suppression of $\mathrm{g}^{(2)}(0)_{\text {fit }}=0.076 \pm 0.015$ at $10 \mathrm{~K}$ and $\mathrm{g}^{(2)}(0)_{\text {fit }}=0.114 \pm 0.022$ at $40 \mathrm{~K}$ 

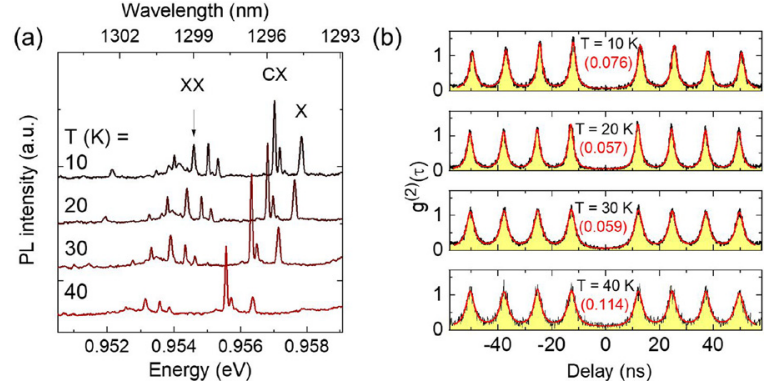

FIG. 2. (a) Temperature-dependent $\mu \mathrm{PL}$ spectra of exciton $(\mathrm{X}, \mathrm{CX})$ and biexciton $(\mathrm{XX})$ complexes recorded under non-resonant $\mathrm{CW}$ excitation at $785 \mathrm{~nm}$. (b) Corresponding $g^{(2)}(\tau)$ histograms and extracted $g^{(2)}(0)$ values (in red) of the CX line.

under pulsed non-resonant wetting-layer excitation ( $974 \mathrm{~nm})$, as shown in Fig. 2(b). The fit presented in Fig. 2(b) is based on a monoexponential decay convoluted with the instrument response function (IRF) of the detection system. The fit yields a background signal of about 0.05 in $\mathrm{g}^{(2)}(\tau)$ due to uncorrelated background emission. Noteworthily, the lifetime of the CX state varies in the investigated temperature range between $\sim 1.6 \mathrm{~ns}$ and $\sim 1.9 \mathrm{~ns}$ with no clear trend although state refilling processes from higher levels, ${ }^{34}$ influencing the occupation per excitation pulse of the CX state, are seen for higher temperatures (see the supplementary material-Fig. S2).

Next, we examined the source brightness in terms of the photonextraction efficiency $\eta_{\text {ext }}$. The QD was excited by pulsed non-resonant $(\sim 860 \mathrm{~nm})$ excitation at saturation of the CX intensity. Under these conditions, we estimate a lower bound of $\eta_{\text {ext }}$ assuming that the internal QD efficiency is close to one (i.e., the non-radiative rate is negligible). The emitted photons were detected by SNSPDs with an overall setup efficiency of $\eta_{\text {setup }} \sim 5.4 \%$. For the given laser pulse repetition rate of $80 \mathrm{MHz}$, we obtained 172.000 counts per seconds (cps) for the sum of $\mathrm{X}$ and CX emission, yielding an extraction efficiency of $\eta_{\text {ext }}=\frac{c p s}{f \eta_{\text {setup }}}=5.0 \%$. This lower bound value is comparable with the expected extraction efficiency of $9.3 \%$ deduced from finiteelement method simulations for this particular mesa (see the supplementary material).

High photon indistinguishability is an important requirement for many applications of SPSs in quantum technology, and it is important to characterize quantum light sources with respect to this figure of merit in order to evaluate their application readiness for advanced quantum technology concepts. The associated TPI effect is measured for photons emitted by the QD-micromesa under study using a fiberbased HOM setup consisting of an unbalanced Mach-Zehnder interferometer (MZI) on the detection side and a complementary MZI on the excitation side. Here, the 4 ns relative optical path delay of the MZI in the detection path is compensated by the temporal separation of excitation pulses induced by the MZI in the excitation path.

Before performing $\mathrm{HOM}$ experiments, we established pulsed quasi-resonant p-shell excitation for the QD-micromesa under study to reduce charge noise and detrimental dephasing processes related to carrier relaxation from higher levels, presumably taking place mainly in the SRL. s-p energy splitting is determined via $\mu$ PLE experiments. As observed in Fig. 3(a), the excitation laser is in resonance with the p-shell of QDs at $1.0225 \mathrm{eV}$. The corresponding s-p splitting amounts to $\Delta E_{\mathrm{s}-\mathrm{p}}=65.7 \mathrm{meV}$ (also see the supplementary material-Fig. S3) and is in good agreement with values reported in Refs. 4 and 35. When exciting quasi-resonantly at an excitation power required to achieve $\sim 70 \%$ of the maximum CX intensity, we achieved a further improvement of the $g^{(2)}(0)$ value. In Fig. $3(\mathrm{~b})$, we fit the correlation data using again a monoexponential decay convoluted with the IRF of the setup and with an uncorrelated background of 0.023 , which yields $\mathrm{g}^{(2)}(0)_{\text {fit }}=0.027 \pm 0.005$ for the telecom O-band QD-micromesa. In addition, we observe noticeable blinking in the side maxima up to time delays $\tau$ of about $40 \mathrm{~ns}$, which we attribute to the memory effect for the subsequent pulses due to local charge fluctuations. ${ }^{36}$ Within $\pm 3 \mathrm{~ns}$ around zero delay, a slight recapture process is also indicated by a minimum observed at $\tau=0$. Noteworthily, the achieved $g^{(2)}(0)$ value of $0.027 \pm 0.005$ is similar to the lowest value $\left[\mathrm{g}^{(2)}(0)=0.03\right]$ observed previously for cavity-coupled InGaAs/GaAs QDs emitting in the telecom O-band ${ }^{4}$ and proves that our advanced device, processed in multiple steps, maintains a single-photon emission with high suppression of multi-photon events. Further improvements are expected for twophoton resonant excitation schemes. ${ }^{37,38}$

In Figs. 3(c) and 3(d), we present TPI histograms obtained for the CX line under p-shell excitation. As we expect an overlap of the side peaks of subsequent laser pulses, it is convenient both to measure the interference histogram for co- and cross-polarized photons and then to quantify the central peak suppression by direct comparison. ${ }^{39}$ According to the fitting function based on a series of Lorentzian peak functions, the histogram in Fig. 3(d) shows a single peak centered at $\tau=0$ with 2.693 coincidences for cross-polarized photons. In the case of co-polarized photons in Fig. 3(c), we observe a suppression of
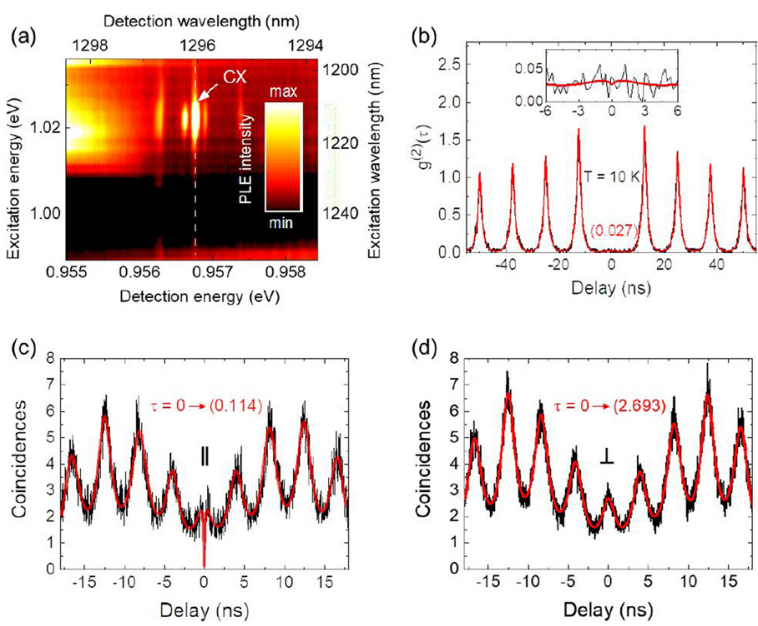

FIG. 3. (a) $\mu \mathrm{PLE}$ data measured on the deterministic QD-micromesa demonstrating spectral resonance with the p-shell at $1.022 \mathrm{eV}$ for excitonic states, i.e., CX emitting at $0.956 \mathrm{eV}$ as indicated by the dashed line. (b) Corresponding pulsed secondorder photon autocorrelation measurement at $10 \mathrm{~K}$, showing clean single-photon emission with $\mathrm{g}^{(2)}(0)=0.027 \pm 0.005$ according to the fitting function (red) superimposed to the raw histogram data. (c) and (d) Two-photon interference histograms measured for the CX emission under pulsed p-shell excitation for co- and crosspolarized configurations, respectively. The HOM effect is proven by the highly reduced coincidences in (c) compared to (d). The red trace corresponds to a fit function used to evaluate the two-photon interference visibility (see the supplementary material for details). 
coincidences down to 0.114. These numbers yield a post-selected TPI visibility $V=\left(g^{(2)}(0)_{\perp}-g^{(2)}(0)_{\|}\right) / g^{(2)}(0)_{\perp}$ of $(96 \pm 10) \%$. Taking into account the integrated peak areas, we obtain a visibility of $(12 \pm 4) \%$ without post-selection. Moreover, by using the post-selected $V$ as an input parameter for a description based on monoexponential decay functions ${ }^{8}$ convoluted with the IRF, we obtain a coherence time of $\tau_{c}=(212 \pm 25)$ ps (see the supplementary material for details). These results show that further optimization and research are required to strongly improve the coherence time and the indistinguishability, especially because advanced quantum applications such as the implementation of fiber-based quantum repeater networks relying on entanglement distribution via Bell-state measurements require high non-post-selected indistinguishability. Most probably, the experimental values achieved here are limited by structural imperfections and charge noise introduced by the SRL, and HOM studies can be used as a sensitive tool in the technological optimization of QDs emitting at wavelengths around $1.3 \mu \mathrm{m}$. Noteworthily, our results for QD-micromesas feature significantly higher postselected indistinguishability and coherence time than $(67 \pm 1) \%$ and $(150 \pm 29)$ ps and similar non-post-selected indistinguishability [(18 \pm 1$) \%]$ as reported previously for a non-resonantly driven $1.3 \mu \mathrm{m}$ QDs coupled to a PhC nanocavity. ${ }^{8}$ Moreover, our photonic structures support broadband enhancement of photon extraction and are very robust, which makes them suitable for the development of stand-alone SPSs.

In summary, we have shown a deterministically fabricated singlephoton device emitting in the telecom O-band. The single-QD micromesa with a backside gold-mirror features single-photon emission stable up to $40 \mathrm{~K}$, which makes it compatible with Stirling cryocoolers for stand-alone operation. The device shows strong multi-photon suppression associated with $\mathrm{g}^{(2)}(0)$ as low as 0.027 under pulsed p-shell excitation. The generated photons show a post-selected indistinguishability of $(96 \pm 10) \%$ and an application-relevant indistinguishability of $(12 \pm 4) \%$ without post-selection. The broadband design of the microstructure facilitates a photon-extraction efficiency of $\sim 5 \%$. This value is well comparable to the simulated extraction efficiency of about $9 \%$. The developed flip-chip based-telecom-SPS device technology is compatible with spectral strain tuning when combined with a lower piezo-actuator ${ }^{40}$ and with circular Bragg resonator SPSs, which promises significantly higher photon-extraction efficiency in the future. ${ }^{41}$ In summary, we developed and deterministically fabricated a QD-SPS emitting in the telecom O-band, which meets important requirements for single-photon sources for application in fiber-based quantum communication. Combining these requirements, especially for emission in the O-band, is a significant step toward versatile high-performance single-photon sources. However, in light of the $12 \%$ indistinguishability, it is clear that further structural optimization is required to meet the stringent requirements of advanced quantum photonic applications in terms of the on-demand generation of single photons with high photon indistinguishability.

See the supplementary material for information on the identification of excitonic complexes in quantum dots, the emission dynamics and spontaneous emission lifetime of QDs, microphotoluminescence excitation spectroscopy, and optical studies under quasi-resonant $\mathrm{p}$-shell excitation. Moreover, the details of the numerical optimization of QDmicromesas and the evaluation of Hong-Ou-Mandel experiments are provided.
The research leading to these results received funding from the German Research Foundation through No. CRC 787 "Semiconductor Nanophotonics: Materials, Models, Devices," the Volkswagen Foundation via the project NeuroQNet, and the FI-SEQUR project jointly financed by the European Regional Development Fund (EFRE) of the European Union in the framework of the program to promote research, innovation, and technologies (Pro FIT). T.H. gratefully acknowledges financial support of the German Federal Ministry of Education and Research (BMBF) via the project "QuSecure" (Grant No. 13N14876) within the funding program Photonic Research Germany. P.M. gratefully acknowledges financial support from the Polish Ministry of Science and Higher Education within the "Mobilność Plus-V edycja” program.

\section{DATA AVAILABILITY}

The data that support the findings of this study are available from the corresponding author upon reasonable request.

\section{REFERENCES}

${ }^{1}$ E. Knill, R. Laflamme, and G. J. Milburn, Nature 409, 46 (2001).

2J. L. O’Brien, A. Furusawa, and J. Vučković, Nat. Photonics 3, 687 (2009).

${ }^{3}$ C. H. Bennet and G. Brassard, in Proceedings of the IEEE International Conference on Computer System Signal Processing (1984), p. 175

${ }^{4}$ Ł. Dusanowski, P. Holewa, A. Maryński, A. Musiał, T. Heuser, N. Srocka, D. Quandt, A. Strittmatter, S. Rodt, J. Misiewicz, and S. Reitzenstein, Opt. Express 25, 31122 (2017)

${ }^{5}$ F. Olbrich, J. Höschele, M. Müller, J. Kettler, S. Luca Portalupi, M. Paul, M. Jetter, and P. Michler, Appl. Phys. Lett. 111, 133106 (2017).

${ }^{6}$ Ł. Dusanowski, M. Syperek, P. Mrowiński, W. Rudno-Rudziński, J. Misiewicz, A. Somers, S. Höfling, M. Kamp, J. P. Reithmaier, and G. Sęk, Appl. Phys. Lett. 105, 021909 (2014).

${ }^{7}$ M. B. Ward, O. Z. Karimov, D. C. Unitt, Z. L. Yuan, P. See, D. G. Gevaux, A. J. Shields, P. Atkinson, and D. A. Ritchie, Appl. Phys. Lett. 86, 201111 (2005).

${ }^{8}$ J. Kim, T. Cai, C. J. K. Richardson, R. P. Leavitt, and E. Waks, Optica 3, 577 (2016).

${ }^{9}$ A. Fiore, C. Zinoni, B. Alloing, C. Monat, L. Balet, L. H. Li, N. Le Thomas, R. Houdré, L. Lunghi, M. Francardi, A. Gerardino, and G. Patriarche, J. Phys. 19, 225005 (2007)

${ }^{10}$ P. Mrowiński, A. Musiał, K. Gawarecki, Ł. Dusanowski, T. Heuser, N. Srocka, D. Quandt, A. Strittmatter, S. Rodt, S. Reitzenstein, and G. Sęk, Phys. Rev. B 100, 115310 (2019).

${ }^{11}$ E. Goldmann, S. Barthel, M. Florian, K. Schuh, and F. Jahnke, Appl. Phys. Lett. 103, 242102 (2013).

${ }^{12}$ L. Seravalli, G. Trevisi, P. Frigeri, D. Rivas, G. Muñoz-Matutano, I. Suárez, B. Alén, J. Canet, and J. P. Martínez-Pastor, Appl. Phys. Lett. 98, 173112 (2011).

${ }^{13}$ M. Paul, F. Olbrich, J. Höschele, S. Schreier, J. Kettler, S. L. Portalupi, M. Jetter, and P. Michler, Appl. Phys. Lett. 111, 033102 (2017).

${ }^{14}$ T. Miyazawa, K. Takemoto, Y. Nambu, S. Miki, T. Yamashita, H. Terai, M. Fujiwara, M. Sasaki, Y. Sakuma, M. Takatsu, T. Yamamoto, and Y. Arakawa, Appl. Phys. Lett. 109, 132106 (2016).

${ }^{15}$ P. Mrowiński, Ł. Dusanowski, A. Somers, S. Höfling, J. P. Reithmaier, J. Misiewicz, and G. Sęk, Acta Phys. Pol. A 132, 382 (2017).

${ }^{16}$ Ł. Dusanowski, M. Syperek, J. Misiewicz, A. Somers, S. Höfling, M. Kamp, J. P. Reithmaier, and G. Sęk, Appl. Phys. Lett. 108, 163108 (2016).

${ }^{17}$ M. Sartison, L. Engel, S. Kolatschek, F. Olbrich, C. Nawrath, S. Hepp, M. Jetter, P. Michler, and S. L. Portalupi, Appl. Phys. Lett. 113, 032103 (2018).

${ }^{18}$ K. Takemoto, M. Takatsu, S. Hirose, N. Yokoyama, Y. Sakuma, T. Usuki, T. Miyazawa, and Y. Arakawa, J. Appl. Phys. 101, 081720 (2007).

${ }^{19}$ M. Benyoucef, M. Yacob, J. P. Reithmaier, J. Kettler, and P. Michler, Appl. Phys. Lett. 103, 162101 (2013). 
${ }^{20}$ J. Kettler, M. Paul, F. Olbrich, K. Zeuner, M. Jetter, P. Michler, M. Florian, C. Carmesin, and F. Jahnke, Phys. Rev. B 94, 045303 (2016).

${ }^{21}$ C. Nawrath, F. Olbrich, M. Paul, S. L. Portalupi, M. Jetter, and P. Michler, Appl. Phys. Lett. 115, 023103 (2019).

${ }^{22}$ K. Nishi, H. Saito, S. Sugou, and J. S. Lee, Appl. Phys. Lett. 74, 1111 (1999).

${ }^{23}$ V. M. Ustinov, N. A. Maleev, A. E. Zhukov, A. R. Kovsh, A. Y. Egorov, A. V. Lunev, B. V. Volovik, I. L. Krestnikov, Y. G. Musikhin, N. A. Bert, P. S. Kop'ev, Z. I. Alferov, N. N. Ledentsov, and D. Bimberg, Appl. Phys. Lett. 74, 2815 (1999).

${ }^{24}$ E. Goldmann, M. Paul, F. F. Krause, K. Mueller, J. Kettler, T. Mehrtens, A. Rosenauer, M. Jetter, P. Michler, and F. Jahnke, Appl. Phys. Lett. 105, 152102 (2014).

${ }^{25}$ M. Gschrey, R. Schmidt, J.-H. Schulze, A. Strittmatter, S. Rodt, and S. Reitzenstein, J. Vac. Sci. Technol., B 33, 021603 (2015).

${ }^{26}$ R. Trotta, P. Atkinson, J. D. Plumhof, E. Zallo, R. O. Rezaev, S. Kumar, S. Baunack, J. R. Schröter, A. Rastelli, and O. G. Schmidt, Adv. Mater. 24, 2668 (2012).

${ }^{27}$ S. Fischbach, A. Kaganskiy, E. B. Y. Tauscher, F. Gericke, A. Thoma, R. Schmidt, A. Strittmatter, T. Heindel, S. Rodt, and S. Reitzenstein, Appl. Phys. Lett. 111, 011106 (2017)

${ }^{28}$ N. Srocka, A. Musiał, P.-I. Schneider, P. Mrowiński, P. Holewa, S. Burger, D. Quandt, A. Strittmatter, S. Rodt, S. Reitzenstein, and G. Sęk, AIP Adv. 8, 085205 (2018).

${ }^{29}$ P. Mrowiński and G. Sęk, Physica B 562, 141 (2019).

${ }^{30}$ M. Gschrey, F. Gericke, A. Schüßler, R. Schmidt, J.-H. Schulze, T. Heindel, S. Rodt, A. Strittmatter, and S. Reitzenstein, Appl. Phys. Lett. 102, 251113 (2013).

${ }^{31}$ A. Schlehahn, S. Fischbach, R. Schmidt, A. Kaganskiy, A. Strittmatter, S. Rodt,

T. Heindel, and S. Reitzenstein, Sci. Rep. 8, 1340 (2018).
${ }^{32}$ A. Musiał, K. Żołnacz, N. Srocka, O. Kravets, J. Große, J. Olszewski, K. Poturaj, G. Wójcik, P. Mergo, K. Dybka, M. Dyrkacz, M. Dłubek, K. Lauritsen, A. Bülter, P.-I. Schneider, L. Zschiedrich, S. Burger, S. Rodt, W. Urbańczyk, G. Sęk, and S. Reitzenstein, Adv. Quantum Technol. 3, 2000018 (2020).

${ }^{33}$ A. Schlehahn, L. Krüger, M. Gschrey, J.-H. Schulze, S. Rodt, A. Strittmatter, T. Heindel, and S. Reitzenstein, Rev. Sci. Instrum. 86, 013113 (2015).

${ }^{34}$ Ł. Dusanowski, M. Syperek, W. Rudno-Rudziński, P. Mrowiński, G. Sęk, J. Misiewicz, A. Somers, J. P. Reithmaier, S. Höfling, and A. Forchel, Appl. Phys. Lett. 103, 253113 (2013).

${ }^{35}$ P. Podemski, A. Musiał, K. Gawarecki, A. Maryński, P. Gontar, A. Bercha, W. A. Trzeciakowski, N. Srocka, T. Heuser, D. Quandt, A. Strittmatter, S. Rodt, S. Reitzenstein, and G. Sęk, Appl. Phys. Lett. 116, 023102 (2020).

${ }^{36}$ C. Santori, D. Fattal, J. Vučković, G. S. Solomon, E. Waks, and Y. Yamamoto, Phys. Rev. B 69, 205324 (2004).

${ }^{37}$ M. Mueller, S. Bounouar, K. D. Jöns, M. Glassl, and P. Michler, Nat. Photonics 8, 224 (2014).

${ }^{38}$ L. Schweickert, K. D. Jöns, K. D. Zeuner, S. F. Covre Da Silva, H. Huang, T. Lettner, M. Reindl, J. Zichi, R. Trotta, A. Rastelli, and V. Zwiller, Appl. Phys. Lett. 112, 093106 (2018).

${ }^{39}$ A. Thoma, P. Schnauber, M. Gschrey, M. Seifried, J. Wolters, J.-H. H. Schulze, A. Strittmatter, S. Rodt, A. Carmele, A. Knorr, T. Heindel, and S. Reitzenstein, Phys. Rev. Lett. 116, 033601 (2016).

${ }^{40}$ M. Schmidt, M. V. Helversen, S. Fischbach, A. Kaganskiy, R. Schmidt, A. Schliwa, T. Heindel, S. Rodt, and S. Reitzenstein, Opt. Mater. Express 10, 76 (2020).

${ }^{41}$ L. Rickert, T. Kupko, S. Rodt, S. Reitzenstein, and T. Heindel, Opt. Express 27, 36824 (2019) 